\title{
Hyperglycemia and Glasgow Coma Scale in Pediatric Traumatic Brain Injury in the Emergency Room
}

\author{
Article by Karthick Jayapal \\ MBBS, M.D(ped), Fellowship in Pediatric Emergency medicine, Fellowship in Pediatric \\ Trauma, Specialist Pediatric ER, Shri SathyaSai Medical college and Research Institute, \\ India \\ Email: karthickchildcare@gmail.com
}

\begin{abstract}
Introduction: A high blood glucose is common in actually ill neurological patients, even in nondiabetics. A consensus regarding the cut-off blood glucose level that would be related to poor prognosis in children and adolescent with head trauma, which makes the comparison of different studies important. Objective: Prevalence of acute hyperglycemia and Glasgow coma scale (GCS) in Pediatric Traumatic brain injury in the Emergency Room. Method: Prospective cross-sectional study of pediatric head injury patients in the emergency room during one year period. The cut-off value of $150 \mathrm{mg} / \mathrm{dL}$ to define hyperglycemia was considered. Results: A total of 440 children were included and 65 had admission hyperglycemia. Hyperglycemia was present in $11 \%$ of mild head trauma cases; $35 \%$ of those with moderate head trauma and 54\% of severe head trauma. In this study, observed that among children with higher blood glucose levels, 83\% had abnormal findings on cranial computed tomography scans. The prevalence of hyperglycemia is higher in patients with severe head trauma, as well as in those with abnormal findings on CCT scans. Conclusion: Hyperglycemia was more prevalent in severe head trauma (GCS8), regardless of etiology of trauma, mode of injury or multiple trauma in children with abnormal findings on head computed tomography scans.
\end{abstract}

Keywords: adolescents, children, head trauma, hyperglycemia, prevalence

\section{Introduction}

Guidelines for diabetes and classification are widely described ${ }^{1,2}$. A high blood glucose is common in actually ill neurological patients, even in non-diabetics. A consensus regarding the cut-off blood glucose level that would be related to poor prognosis in children and adolescent with head trauma, which makes the comparison of different studies imortant ${ }^{3-9}$.

Some authors believe that patients with hyperglycemia general have a low Glasgow Coma Scale (GCS) score 3-6, poor neurological prognosis based on GCS) and a history of severe trauma with significant brain injury ${ }^{6,7}$. It is well known that such acute hyperglycemia is a result of catecholamine effects $^{8,9}$. Some authors, however, disagree on the association of hyperglycemia and poor prognosis, particularly in children and adolescents, as high blood glucose levels are transient and basically reflect a body response after ijury ${ }^{10}$. In the patient study we aimed to verify the prevalence of acute hyperglycemia in pediatric patients with head trauma, according to the severity of head injury.

Purpose of this study is of great importance in pediatric age group, as more study are related with adult population. Early Hyperglycemia in ER, correlates well with the severity of traumatic brain injury irrespective of the mode of trauma, gives the treating physician as one the important tool in assessment and decision making.

\section{Method}

A prospective cross-sectional study was carried out with information derived from medical records of pediatric patients with head trauma. data collected from medical records included age, sex, mechanism of injury, classification of trauma (isolated head trauma or multiple trauma), GCS score 
and blood glucose level on admission and cranial computed tomography (CCT) scan results. Medical records of patients aged from 0 to 14 years-old with head trauma presented in the emergency room for 1 year.

World Health organization (WHO) definition for pediatric age was used here ${ }^{11}$. Severity of head trauma was accessed by GCS score on admission, and when necessary the modification for use in children under 2 years ${ }^{12-14}$. Findings on the CCT scan considered to be abnormal included any brain lesion such as brain swelling, diffuse axonal injury and intracranial hemorrhages. The CCT scans were done in the same hospital and at the same model of tomography machine.

The results were checked by experienced radiologists and neurosurgeons. Exclusion criteria included a previous diagnosis of diabetes, absence of GCS score, CCT scan or blood glucose levels on the first $24 \mathrm{~h}$ of admission and hospital stay less than $48 \mathrm{~h}$.

Hyperglycemia Blood glucose levels were determined by capillary blood glucose obtained through fingertips' puncture at hospital admission. The results were shown in digital blood glucose monitor. This same technique was used in every patient. We considered values above $150 \mathrm{mg} / \mathrm{dl}$ as hyperglycemia, like other authors have already claimed ${ }^{3}$.

Ethical concerns and data analyses the study was approved by the Hospital ethics Committee under registration no 06/07. Either parents or other legally responsible signed informed consent before inclusion in the study. No extra exams, besides the ones usually performed in an emergency room, were done.

Categorical data were analyzed by using $\chi^{2}$ analyses and Pearson analysis was used to verify correlation between GCS and blood glucose level on admission.

\section{Results}

A total of 440 patients were eligible for inclusion. Mean age was $9.8( \pm 6)$ years; $73.2 \%$ were male. Regarding the type of trauma, the most frequent were fall from a height (34.1\%) and vehiclepedestrian accidents (18.2\%). regarding the GCS classification, 295 (72.1\%) patients had mild head trauma (GCS 13-15), 70 (13.5\%) had moderate head trauma (GCS 9-12), whereas 74(14.4\%) suffered severe head trauma (GCS 3-8). Concerning blood glucose levels on admission, 65 (17.6\%) patients had hyperglycemia. Hyperglycemia was found in $73.3 \%$ of male patients and $55 \%$ of traffic accidents victims.

The prevalence of hyperglycemia increased proportionally to the head trauma severity: $11 \%$ in mild head trauma, $35 \%$ in moderate head trauma ( $\mathrm{p}<0.01$ when compared to mild head trauma) and $54 \%$ in cases considered severe $(\mathrm{p}<0.01$ when compared to mild head trauma). An inverse relationship between admission glucose levels and GCS score was found, using Pearson analysis $(\mathrm{r}=0.32 ; \mathrm{p}=0.01)$.

Regarding the cases of isolated head trauma or multiple traumas, in those with isolated head injury we observed hyperglycemia in $15 \%$ versus $20 \%$ of the other group ( $\mathrm{p}=0.28)$. Brain swelling $(32 \%)$, and intracranial hemorrhages (20\%) were the most important abnormal findings on CCT scan.

Among hyperglycemic patients, $83 \%$ had abnormal brain parenchymal findings. However only $35.6 \%$ of the normoglycemic patients had abnormal brain parenchymal findings according CCT scans results.

\section{Discussion}

In accordance with previous studies ${ }^{15}$, our findings showed the predominance of male sex and falls in pediatric head trauma victims. We did not observe statistical differences in hyperglycemia prevalence regarding sex $(\mathrm{p}=0.98)$ and type of trauma. Regarding the GCS classification, we find a preponderance of mild head trauma victims, which is also widely described in previous studies. It is important to notice that patients with mild head trauma generally stay in hospital for short periods of time, have less severe or no brain damage and do not suffer a meaningful metabolic response related to trauma ${ }^{16}$, so it is not usual to diagnose hyperglycemia in those patients.

Hyperglycemia is indeed more frequently observed in severe head trauma victims and in those that 
suffered multiple trauma, according to some studies ${ }^{3,4,6,17,18}$. The explanation would be that these patients have a more important metabolic response to injury due to the effects of cortisol, glucagon and epinephrine release, causing intracellular acidosis, lactate accumulation, high blood glucose levels and neuronal injury ${ }^{6,19-21}$.

Therefore, our data are in accordance with the results from other authors who correlate the head trauma severity and the metabolic response to trauma, particularly higher blood glucose levels ${ }^{7,22,23}$. Some studies show that hyperglycemia is associated with a worse outcome, regardless of injury characteristics $^{24}$. We did not observe statistically significant differences in blood glucose levels in patients with isolated head injury and in the ones with multiple traumas. The probable cause might be that we considered multiple trauma any kind of injury associated with head trauma, regardless of the degree of injury. We could have overestimated the amount of multiple trauma victims.

The observation of abnormal CCT findings was more frequent in hyperglycemic patients. Such an association was also described by previous studies and possible explanations would be that brain injury evokes a metabolic response that results in hyperglycemia and/or that hyperglycemia itself is responsible for more meaningful brain lesions on CCT scans ${ }^{25,26}$. Our knowledge up to now does not permit to conclude if a high blood glucose level is a mediator or a marker of brain injury.

Inclusion the prevalence of hyperglycemia is higher in patients with severe head trauma, as well as in those with abnormal findings on CCT scans. All these variables are generally associated with significant brain tissue lesion. We cannot conclude if the higher blood glucose levels are responsible for poorer outcomes in the present observation. The meaning of hyperglycemia in the outcomes of children and adolescents with severe head trauma will be analyzed in a future study.

The most common classification system for TBI severity is based on GCS score determined at the time of injury. The GCS is a 3- to 15-point scale used to assess a patient's level of consciousness and level of neurologic functioning. It consists of 3 sections, each of which is scored: best motor response, best verbal response, and eye opening. A total score of 3-8 for the 3 sections indicates severe TBI, a score of 9-12 indicates moderate TBI, and a score of 13-15 indicates mild TBI.

The above data obtained, act as a one of the early significant tool in ER for severity of pediatric traumatic brain injury and decision making even in mild and moderate head trauma. Hyperglycemia correlates well with drop in GCS in accordance with severity of brain injury.

\section{Conclusion}

Hyperglycemia was more prevalent in severe head trauma (Gㅌ), regardless of etiology of trauma, mode of injury or multiple trauma in children with abnormal findings on head computed tomography scans

\section{Acknowledgements}

I would like express my gratitude to my professor Vijayarangan MD for helping me with this study.

\section{References}

[1]. Bochicchio GV, Sung J, Joshi M. Persistent hyperglycemia is predictive of outcome in critically ill trauma patients. J Trauma (2005); 58; 921-924.

[2]. Bochicchio GV, Joshi M, Bochicchio KM. Early hyperglycemic control is important in critically injured trauma patients. J Trauma (2007); 63; 1353-1358.

[3]. Chiaretti A, De BR, Langer A. Prognostic implications of hyperglycaemia in paediatric head injury. Childs NervSyst (1998); 14:455-459.

[4]. Chiaretti A, Piastra M, Pulitano S. Prognostic factors and outcome of children with severe head injury: an 8-year experience. Childs NervSyst (2002); 18:129-136.

[5]. Cochran A, Scaife ER, Hansen KW, Downey EC. Hyperglycemia and outcomes from pediatric traumatic brain injury. J Trauma (2003); 55: 1035-1038.

[6]. Durr JA, Hoffman WH, Sklar AH, Steinhart CM. Correlates of brain edema in uncontrolled IDDM. 
Texila International Journal of Medicine

Volume 4, Issue 2, Dec 2016

Diabetes (1992); 41; 627-632.

[7]. Epstein J, Breslow MJ. The stress response of critical illness. Crit Care Clin (1999); 15; 17-33. 20. Hirsch IB.

[8]. Jeremitsky E, Omert LA, Dunham CM, Wilberger J, Rodriguez A. The impact of hyperglycemia on patients with severe brain injury. J Trauma (2005); 58; 47-50.

[9]. Kuzuya T, Nakagawa S, Satoh J. Report of the Committee on the classification and diagnostic criteria of diabetes mellitus. Diabetes Res ClinPract (2002); 55:65-85.

[10]. Kushner M, Nencini P, Reivich M. Relation of hyperglycemia early in ischemic brain infarction to cerebral anatomy, metabolism, and clinical outcome. Ann Neurol (1990); 28: 129-135.

[11]. Lam AM, Winn HR, Cullen BF, Sundling N. Hyperglycemia and neurological outcome in patients with head injury. J Neurosurg (1991); 75: 545-551.

[12]. Laird AM, Miller PR, Kilgo PD, Meredith JW, Chang MC. Relationship of early hyperglycemia to mortality in trauma patients. J Trauma (2004); 56; 1058-1062.

[13]. Marcoux KK. Management of increased intracranial pressure in the critically ill child with an acute neurological injury. AACN Clin Issues (2005); 16:212-231.

[14]. Melo JR, Santana DL, Pereira JL, Ribeiro TF. [Traumatic brain injury in children and adolescents at Salvador City, Bahia, Brazil]. Arq Neuropsiquiatr (2006); 64:994-996.

[15]. Melo JR, Silva RA, Moreira ED Jr. [Characteristics of patients with head injury at Salvador City (Bahia-Brazil)]. Arq Neuropsiquiatr (2004); 62;711-714.

[16]. In-patient hyperglycemia--are we ready to treat it yet? J ClinEndocrinolMetab (2002);87; 975-977.

[17]. Orliaguet GA, Meyer PG, Baugnon T. Management of critically ill children with traumatic brain injury. PaediatrAnaesth (2008); 18 :455-461.

[18]. Parish RA, Webb KS. Hyperglycemia is not a poor prognostic sign in head-injured children. J Trauma (1988);28:517-519.

[19]. Parejo P, Stahl N, Xu W, Reinstrup P, Ungerstedt U, Nordstrom CH. Cerebral energy metabolism during transient hyperglycemia in patients with severe brain trauma. Intensive Care Med (2003); 29; 544-550.

[20]. Sung J, Bochicchio GV, Joshi M, Bochicchio K, Tracy K, Scalea TM. Admission hyperglycemia is predictive of outcome in critically ill trauma patients. J Trauma (2005); 59:80-83.

[21]. Schelp AO, Burini RC. [Cerebral glucose metabolism in craniocerebral trauma: an evaluation]. Arq Neuropsiquiatr (1995);53:698-705.

[22]. Teasdale G, Jennett B. Assessment of coma and impaired consciousness. A practical scale. Lancet (1974)13; 7872; 81-84.

[23]. Tominaga M. Diagnostic criteria for diabetes mellitus]. RinshoByori (1999); 47:901-908.

[24]. Umpierrez GE, Isaacs SD, Bazargan N, You X, Thaler LM, Kitabchi AE. Hyperglycemia: an independent marker of in-hospital mortality in patients with undiagnosed diabetes. J ClinEndocrinolMetab (2002); 87; 978982.

[25]. Yamaguchi N. Sympathoadrenal system in neuroendocrine control of glucose: mechanisms involved in the liver, pancreas and adrenal gland under hemorrhagic and hypoglycemic stress. Can J PhysiolPharmacol (1992);70:167-206.

[26]. World Health Organization. Child and adolescent health and development. Available at (http:///www.who.int/en/) Accessed November 01, (2007). 